From the authors:

We thank D. Stanescu for his interest in our paper [1] and for giving us the opportunity to clarify an important methodological issue. First of all, we would like it to be remembered that our document was intended for use in designing clinical trials on pharmacological treatments of chronic obstructive pulmonary disease. The point we wanted to make clear is that, because of the well known potential errors inherent to dilution methods and body plethysmography [2-4], they should not be used interchangeably within a clinical trial. We are aware that keeping the panting frequency below $1 \mathrm{~Hz}$ helps reduce overestimation of lung volumes measured by body plethysmography in the presence of airflow obstruction $[5,6]$, but this has to be confirmed in very severe chronic obstructive pulmonary disease and it may not be easily feasible in large multicentre clinical trials. Notwithstanding, we agree that, whenever possible, the plethysmographic method should be adopted, not only because it is less time consuming, but also because it allows assessment of repeatability of measurements within sessions and, thus, better quality control.

\section{Cazzola*, V. Brusasco" ${ }^{\#}$ and F.J. Martinez}

*University of Rome Tor Vergata, Internal Medicine, Unit of Respiratory Diseases, Rome, and "Dept of Internal Medicine, University of Genoa, Genova, Italy. "Dept of Internal Medicine, University of Michigan Health-System, Ann Arbor, MI, USA.

\section{STATEMENT OF INTEREST}

Statements of interest for M. Cazzola and V. Brusasco can be found at www.erj.ersjournals.com/misc/statements.shtml

\section{REFERENCES}

1 Cazzola M, MacNee W, Martinez FJ, et al. Outcomes for COPD pharmacological trials: from lung function to biomarkers. Eur Respir J 2008; 31: 416-469.

2 Mitchell MM, Renzetti AD Jr. Evaluation of a single-breath method of measuring total lung capacity. Am Rev Respir Dis 1968; 97: 571-580.

3 Mikus JP, Robins AG, Burke GW, Lyons LD, Epler GR, Smith AA. Comparison of 5 methods for determination of FRC and TLC in 100 persons: appendix 6 of the American Thoracic Society Epidemiology Standardization Project. Am Rev Respir Dis 1978; 118: 92-104.

4 Rodenstein DO, Stanescu DC. Reassessment of lung volume measurement by helium dilution and by body plethysmography in chronic air-flow obstruction. Am Rev Respir Dis 1982; 126: 1040-1044.

5 Rodenstein DO, Stanescu DC, Francis C. Demonstration of failure of body plethysmography in airway obstruction. J Appl Physiol 1982; 52: 949-954.

6 Rodenstein DO, Stanescu DC. Frequency dependence of plethysmographic volume in healthy and asthmatic subjects. J Appl Physiol 1983; 54: 159-165.

DOI: $10.1183 / 09031936.00056808$

\title{
Improvement with long-term itraconazole therapy for Fonsecaea pedrosoi-related mediastinal phaeohyphomycosis
}

\section{To the Editors:}

In the September 2006 issue of the European Respiratory Journal, we reported the first culture-proven case of mediastinal mass due to Fonsecaea pedrosoi that had been successfully managed medically [1]. Maintenance therapy with oral itraconazole $100 \mathrm{mg}$ b.i.d. was continued for a total of 3 yrs. This decision was taken in view of the rarity of the disease, paucity of data on the management of such cases and good clinico-radiological response to 6 months of therapy with this antifungal agent.

During this time period, the patient continued to experience clinical improvement, in the form of complete resolution of the dyspnoea and dysphagia that had mandated tracheostomy and feeding jejunostomy, respectively, at the time of initial presentation. Hoarseness of voice had also improved significantly with speech therapy. The patient is now able to carry out all activities of daily living and has restarted his professional work. The patient was assessed with repeat computed tomography (CT) scans of the thorax at 1-yr intervals. A significant regression in the size of the mediastinal mass was also observed on CT (fig. 1). In view of the patient's clinical and radiological stability, itraconazole treatment has now been stopped and a close follow-up planned.

The authors' aim behind this communication is only to stress the fact that long-term itraconazole therapy may help to achieve sustained improvement followed by stability in both clinical symptoms and radiological lesions of patients with this rare entity. 

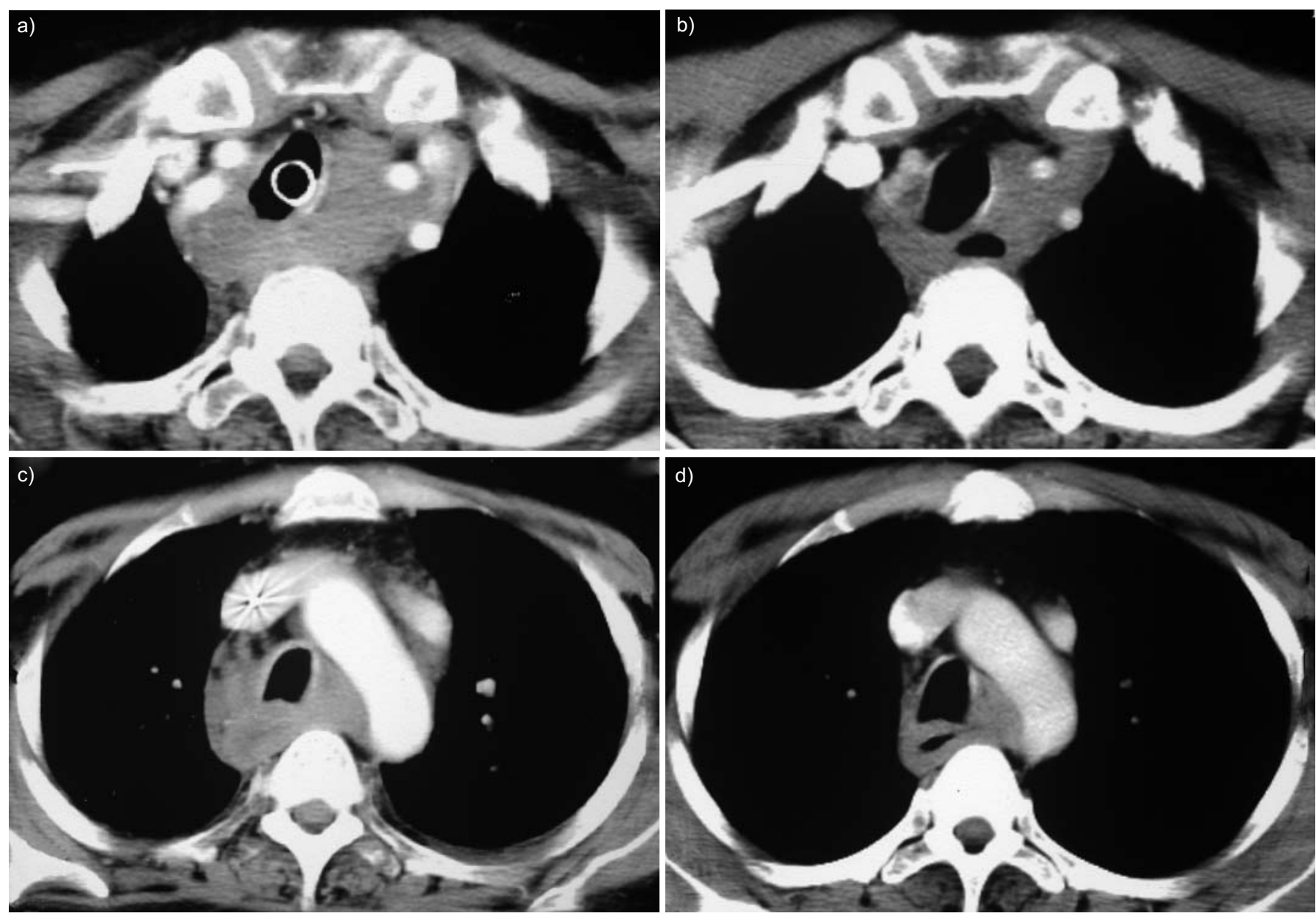

FIGURE 1. a, c) The pre-treatment (baseline) images obtained at the level just below the thoracic inlet and those at the level of aortic arch. b, d) Post-treatment images. A substantial reduction in the size of the mediastinal mass can be seen.

\section{N. Singh, R. Agarwal and D. Gupta}

Dept of Pulmonary Medicine, Postgraduate Institute of Medical Education and Research (PGIMER), Chandigarh, India.

\section{REFERENCES}

1 Singh N, Agarwal R, Gupta D, Shivaprakash MR, Chakrabarti A. An unusual case of mediastinal mass due to Fonsecaea pedrosoi. Eur Respir J 2006; 28: 662-664.

\section{STATEMENT OF INTEREST}

None declared.

\section{The Leicester Cough Monitor: a semi-automated, semi-validated cough detection system?}

To the Editors:

Objective cough monitoring is an important development in the assessment of cough, but it is technically challenging. It is essential that systems designed to automatically detect cough are carefully validated. The recent study by BIRRING et al. [1] reports "the validation of the LCM and preliminary findings of 6- and 24-h recordings in patients with chronic cough". One could be forgiven for imagining that this device has been validated for use over a 24-h period. However, this is not the case.

Thorough reading reveals that two validation stages were performed over a period of 2 and $6 \mathrm{~h}$ in a sub-group of subjects. The high sensitivity and specificity refer only to the 2-h analysis. 